\title{
Computational modelling of foot orthosis for midfoot arthritis: a Taguchi approach for design optimization
}

\author{
HAOWEI ZHANG ${ }^{1 \dagger}$, MIKO LIN LV ${ }^{1,2 \dagger}$, JUNYAN YANG ${ }^{1}$, WENXIN NIU ${ }^{3}$,

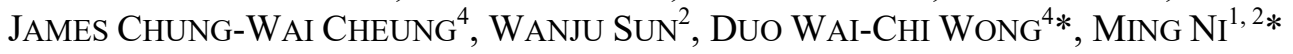 \\ ${ }^{1}$ School of Medical Instrument and Food Engineering, University of Shanghai for Science and Technology, Shanghai, China. \\ ${ }^{2}$ Department of Orthopaedics, Shanghai Pudong New Area People's Hospital, \\ Shanghai University of Medicine and Health Sciences, Shanghai, China. \\ ${ }^{3}$ Yangzhi Rehabilitation Hospital, Sunshine Rehabilitation Centre, Tongji University School of Medicine, Shanghai, China. \\ ${ }^{4}$ Department of Biomedical Engineering, Faculty of Engineering, The Hong Kong Polytechnic University, Hong Kong, China.
}

\begin{abstract}
Purpose: Evaluation of the internal biomechanics of the foot-and-ankle complex is challenging for the prescription of orthosis particularly for midfoot arthritis patients in which the joint condition is crucial. Methods: Using computational modeling and design optimization techniques, the objective of this study was to compare the biomechanical functions among different combinations of design factors using computer simulation. A finite element foot model was reconstructed from a midfoot arthritis patient. Orthotic designs with 3 levels for each of the 3 design factors (arch height, lateral wedge angle, and insole stiffness) contributed to 9 configurations using a fractional factorial design were tested. Results: An increase in peak plantar stress of the midfoot was facilitated by a medium arch height and wedge angle, and stiffest insole material, notwithstanding the combination neither reduced the peak plantar stress of other foot regions nor was consistent with the combination that minimized the stress of the articular cartilage. Conclusions: Insole with high $\operatorname{arch}(H=30 \mathrm{~mm})$, low stiffness $(E=1.0 \mathrm{MPa})$, and medium wedge angle $\left(A=5^{\circ}\right)$ could minimize the stress of the cartilage at the arthritic joint (primary outcome) and could be beneficial to the patients. Also, insole stiffness predominantly influenced cartilage stress. However, secondary outcomes including the stress of the navicular and medial cuneiform and the regional plantar stress did not produce the same solution. Future studies can consider a patient-specific loading profile to further the investigation on the stabilizing effect and the attenuation of load transfer induced by the insole.
\end{abstract}

Key words: finite element simulation, foot and ankle model, posttraumatic arthritis, cuneonavicular joint

\section{Introduction}

Midfoot arthritis is a disabling condition of the midtarsal and tarsometatarsal joints affecting $12 \%$ of older adults and results in pain and functional disability [1]. Patients experience pain during standing and walking and the pain worsens during stair-climbing and walking on uneven terrains [1], [2]. Arthritis would progress to the collapse of the medial longitudinal arch, leading to secondary problems or deformities, such as rigid flatfoot deformity, forefoot abduction/ varus [1], [2]. Eventually, the formation of osteophyte within the joint further deteriorates the arthritic condition that exaggerates pain, swelling, and shoe-wear difficulty [2]. Post-traumatic arthritis of the midfoot is the most common etiology whereas primary degenerative arthritis is linked to the history of injury of the patients [2]. Beside inflammatory and neuropathic arthritis that affect multiple joints, the fusion of the hindfoot joint could lead to secondary arthritis of the midfoot because of the alteration of load transfer and the elevated stress at the midfoot joints [2].

Non-operative interventions for midfoot arthritis are prioritized unless the patients experience high levels of symptoms and restrictions [1]. Regarding the con-

\footnotetext{
* Corresponding authors: Duo Wai-Chi Wong, Department of Biomedical Engineering, Faculty of Engineering, The Hong Kong Polytechnic University, 11 Yuk Choi Road, Hung Hom, Kowloon, Hong Kong 999077, China. Phone: +852 2766-7669, e-mail: duo.wong@polyu.edu.hk; Ming Ni, Department of Orthopaedics, Shanghai Pudong New Area People's Hospital, Shanghai University of Medicine and Health Sciences, No. 349, South Chuanhuan Road, Pudong New Area, Shanghai, 201299, China. Phone: +86 21-20509048, e-mail: gendianqing@163.com

Received: July 7th, 2020

Accepted for publication: September 16th, 2020
} 
servative measures, in addition to analgesics, foot orthoses can alleviate pain and improve functions by reducing midfoot load, controlling rearfoot eversion, redistributing plantar pressure, and supporting the medial longitudinal arch [3], [4]. Rao et al. [3] suggested that full-length foot orthosis decreased the dorsiflexion of the first metatarsophalangeal joint and the range of plantarflexion of the first metatarsal, while the functional foot orthoses could reduce pain and improve functional scores despite the increase of maximum plantar force under the midfoot [5]. Besides, Rao et al. [4] indicated that the custom-molded threequarter-length and full-length foot orthoses, that were both used to treat midfoot arthritis produced significantly different average plantar pressure and contact time of the medial midfoot. To put it briefly, different orthotic designs could present different biomechanical functions and hence effectiveness in treatment. However, there were very few studies that evaluated and compared orthotic designs for midfoot arthritis.

Investigations on the biomechanics of midfoot arthritis have been challenging, particularly under orthotic conditions. Midfoot arthritis patients feature a medial arch collapse and manifest an increase of midfoot plantar loading. It is surprising and sounds implausible that foot orthosis headed for additional midfoot loading to alleviate pain [4], [5]. In fact, the increase of midfoot pressure is intuitive because the contoured and elevated shape the foot orthosis (i.e., arch support) compressed the foot arch region. It shall be noted that the plantar pressure or plantar loading of the midfoot serves as an indicator to support the medial longitudinal arch and to redistribute the high plantar loading from other plantar foot regions, which may not directly be related to the primary problem at the arthritic joint. Assessing the biomechanical environment at the arthritic joint internally remains invasive and thus infeasible in some clinical studies otherwise achieved by cadaveric experiments but often focused on the evaluation of joint fusion procedures. Computer models/simulations, finite element (FE) analysis, can provide a versatile alternative to investigate the internal biomechanical environment and performance of the foot-and-ankle complex which were readily used in the evaluation of pathologies, surgical and non-surgical interventions [6], [7].

To this end, the goal of this study was to compare the influence of different foot orthotic designs (insoles) on the biomechanics of the midfoot-arthritic foot. Particularly, we aimed to evaluate the maximum von Mises stress of the navicular, medial cuneiform, articular cartilage at the arthritic joint (medial cuneonavicular joint), and the plantar foot (heel, midfoot, and metatarsal regions) among nine configurations of foot orthosis involving differences in arch heights, lateral wedge angles at midfoot, and insole stiffness. The investigation was conducted using a FE model reconstructed from a midfoot arthritis patient and simulated with balanced standing and midstance instant during walking.

\section{Materials and methods}

\subsection{Patient information}

The male patient was 18 years old, $175 \mathrm{~cm}$ tall, and weighed $70 \mathrm{~kg}$. He was admitted to the Shanghai Pudong New Area People's Hospital on 14 Dec, 2017 because of the pain of the right foot. He reported no history of trauma and surgery on his right foot. His CT data revealed an uneven joint surface, narrowing of joint space, and sclerosis at the medial cuneonavicular joint. He was diagnosed with midfoot (medial cuneonavicular joint) arthritis with a mild level of medial longitudinal arch collapse. The study was approved by the Ethics Committee of Shanghai Pudong New Area Peoples' Hospital (Reference No.: 2019-16). The patient had consented to participate in this study and agreed to contribute his clinical data for research purposes.

\subsection{Model reconstruction}

\subsubsection{Foot model}

Computed Tomography (CT, GE CT750 High Definition) of the left lower limb was taken on the patient at $1-\mathrm{mm}$ slice interval and $0.5-\mathrm{mm}$ pixel size. During the scan, the patient wore a foot-ankle brace to position the ankle joint at a neutral position with minimum compression on the plantar soft tissue. The images were processed by the software, Mimics 15.0 (Materialise, Leuven, Belgium) to segment and reconstruct the geometry. As shown in Fig. 1, the three-dimensional geometry model consisted of the encapsulated soft tissue and 26 bony segments including the distal tibia, distal fibula, talus, calcaneus, navicular, cuboid, 3 cuneiforms, 5 metatarsals, and 12 phalanges. The geometry of the cartilage at the arthritic site (medial cuneonavicular joint) was also reconstructed from the $\mathrm{CT}$ image, while the other cartilage components were realized by the encapsulated soft tissue which filled up the joint space. The geometry of the articular cartilage at the arthritic site was fine-tuned using the soft 
ware Pro-Engineering 5.0 (PTC, Boston, MA, USA) and SolidWorks (SolidWorks Inc., Dassault Systèmes, Vélizy-Villacoublay, France). The geometry of the arthritic

\section{(a)}

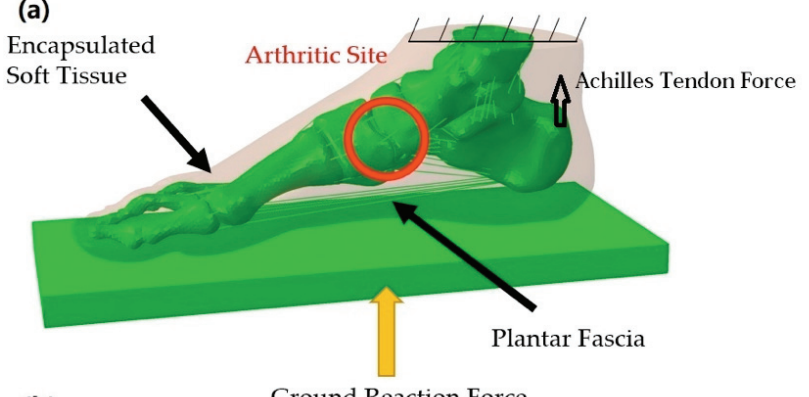

(b)

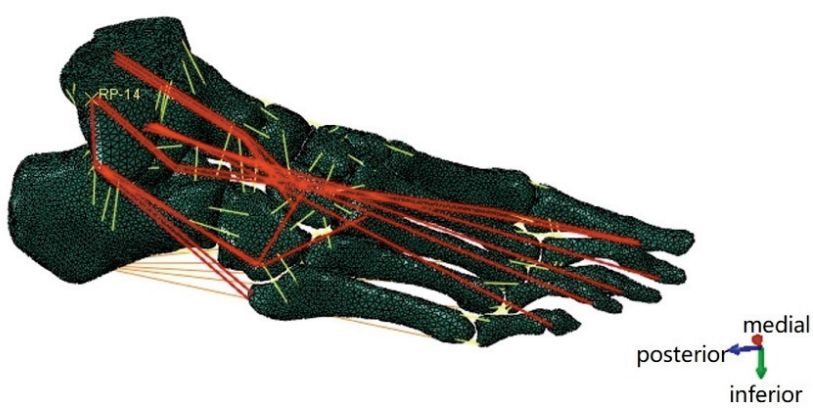

Fig. 1. Geometry of the finite element foot model: (a) foot model illustrating the bone, plantar fascia, encapsulated soft tissue and the arthritic site. The ground reaction force was applied beneath the ground plate whilst the proximal end of the distal tibia and fibula were fixed. Achilles tendon force was also applied at the insertion; (b) foot model without displaying the encapsulated soft tissue. The yellow wires denoted ligaments and plantar fascia and the red wires (connectors)

denoted musculotendon units. Muscle forces were applied through concentric forces at the connectors

cartilage was irregular with an approximated mediolateral width, superoinferior width, and thickness of $17.76 \mathrm{~mm}$, $27.1 \mathrm{~mm}$, and 1.33 to $4.24 \mathrm{~mm}$, respectively.

The ligament and plantar fascia bundles were modeled by truss units linking the relevant anatomical landmarks guided by an orthopaedic surgeon, whereas the major muscle groups, including the tibialis posterior, flexor digitorum longus, flexor hallucis longus, peroneal muscle group, and triceps surae (via Achilles tendon) were modeled by connectors.

\subsubsection{Orthosis (insole) model}

The baseline profile of the orthosis was a total contact insole and was reconstructed using the software SolidWorks. Firstly, as shown in Fig. 2b, the exterior boundary of the plantar foot was extracted to

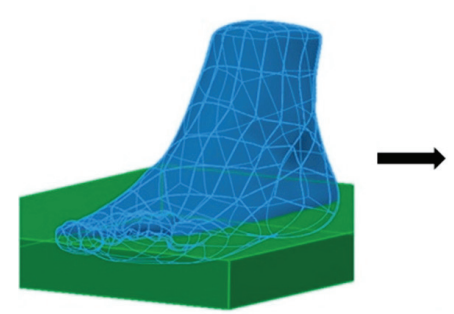

(a)

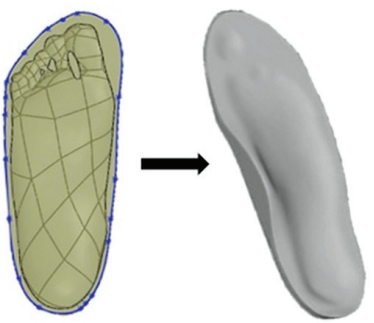

(b) (c)

Fig. 2. Procedures to model the baseline profile of the total contact insole: (a) extrusion of insole block from the exterior boundary of the plantar foot profile

b) the insole block was subtracted by the plantar surface using the Boolean operation,

(c) reconstructed total contact insole

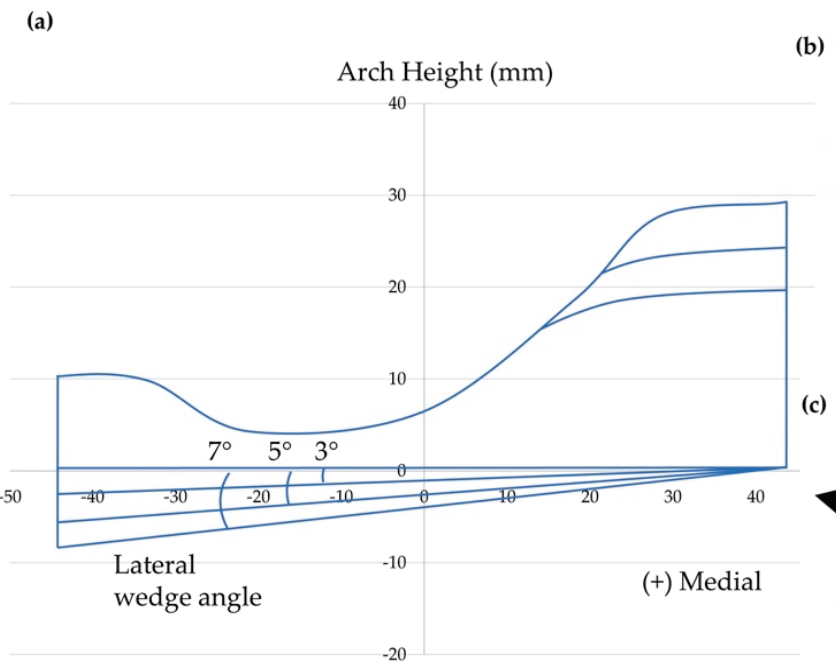

(b)
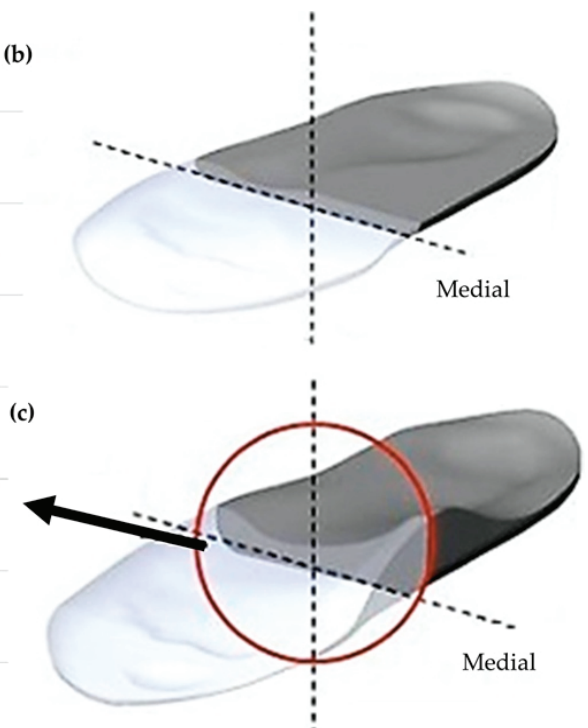

Fig. 3. Adjustment of arch heights and lateral wedge angles to produce different combinations of insole designs:

(a) the cross-section of the insole at the midfoot demonstrating differences in arch height and wedge angle,

(b) illustration of the insole with low arch height, (c) illustration of the insole with high arch height and the location of the cross-section in (a) 
extrude a block. Secondary, the block was subtracted by the plantar surface using the Boolean operators to form a total contact insole. Based on the reconstructed total contact insole, the arch heights and lateral wedge angles at the midfoot were adjusted to produce different combinations of insole configurations, as shown in Fig. 3. There were total of 9 insole configurations fractionally crossed by 3 independent factors, including the arch height $(A)$, wedge angle $(W)$, and insole stiffness $(E)$, as shown in Table 1. Each of the independent design factors of insoles consisted of 3 levels. The choice of the dimension and stiffness of the insole was adopted from an existing study and was believed to be commonly used [8].

Table 1. The levels of the independent design factors of the insole including the arch height, lateral wedge angle and insole stiffness

\begin{tabular}{|c|c|c|c|}
\hline Level & $\begin{array}{c}\text { Arch height, } H \\
{[\mathrm{~mm}]}\end{array}$ & $\begin{array}{c}\text { Wedge Angle, } A \\
{\left[{ }^{\circ}\right]}\end{array}$ & $\begin{array}{c}\text { Insole Stiffness, } E \\
{[\mathrm{MPa}]}\end{array}$ \\
\hline 1 & 25 & 3 & 1.5 \\
\hline 2 & 20 & 7 & 1.0 \\
\hline 3 & 30 & 5 & 2.5 \\
\hline
\end{tabular}

\subsection{Mesh creation}

Mesh creation was conducted using the software, Hypermesh 13.0 (Altair, Troy, USA). The bones, cartilage, and the encapsulated soft tissue were meshed with 4-node tetrahedron elements (C3D4), whereas the plantar fascia and the ligaments were meshed as 2-node truss units (T3D2). We assigned an overall mesh size of $2 \mathrm{~mm}$ for the bone and the encapsulated soft tissue. The cartilage at the arthritic site was assigned with a mesh size of $1 \mathrm{~mm}$. The mesh quality was verified by the Element Quality Check module of the Hypermesh software. Although we did not conduct a mesh convergence test, we adopted a more conservative mesh size compared to an existing simulation research which passed the mesh convergence test [9]. There were a total of 86,896 and 459,815 of nodes and elements in the foot model. On the other hand, the mesh size of the 9 insoles is $5 \mathrm{~mm}$ producing the number of elements with a range from 8,405 to 11,305 .

\subsection{Material property}

The material property of the bone was assumed homogeneous and linearly elastic with the Young's modulus and Poisson's ratio of $7.300 \mathrm{MPa}$ and 0.3, respectively [10]. The articular cartilage at the arthritic site was assumed with the Young's modulus of $1 \mathrm{MPa}$ and Poisson's ratio of 0.4 [11]. The encapsulated soft tissue (with the normal cartilage) was assigned with a hyperelastic material property using a second order polynomial strain energy potential equation using coefficients, $C_{10}=0.08556 \mathrm{Nmm}^{-2}, C_{01}=$ $-0.05841 \mathrm{Nmm}^{-2}, C_{20}=0.039 \mathrm{Nmm}^{-2}, C_{11}=$ $-0.02319 \mathrm{Nmm}^{-2}, C_{02}=0.00851 \mathrm{Nmm}^{-2}, D_{1}=$ $3.65273 \mathrm{~mm}^{2} \mathrm{~N}^{-1}[12]$. Both plantar fascia and ligaments were modeled as tension-only truss units with the assigned Young's modulus $350 \mathrm{MPa}$ and $260 \mathrm{MPa}$, respectively [13], [14]. In addition, their cross-sectional area were $58.6 \mathrm{~mm}^{2}$ and $18.4 \mathrm{~mm}^{2}$, respectively [13], [14]. The ground plate was assigned with a relative rigid stiffness (Young's modulus $=17.000 \mathrm{MPa}$, Poisson's ratio $=0.1)[8]$.

\subsection{Boundary and loading conditions}

The midstance instant (identified as 430 milliseconds from the initial contact) during walking was simulated whereas another simulation on balanced standing was also conducted for validation. The proximal end of the tibia, fibula, and the encapsulated soft tissue was fixed for both scenarios and all simulation conditions. For the balanced standing simulation, a vertical ground reaction force of $350 \mathrm{~N}$ (50\% of body weight) was applied beneath the ground plate superiorly, in addition to the Achilles tendon force of $262.5 \mathrm{~N}$ (75\% of the weight on foot) [15]. For the simulation of walking midstance, the vertical ground reaction force and the Achilles tendon force were $600 \mathrm{~N}$ and $640 \mathrm{~N}$, respectively [16]. Muscle forces were applied through concentric load on the reconstructed connectors. The muscle forces of the tibialis posterior, peroneal muscle group, flexor digitorum longus, and flexor hallucis longus were $50 \mathrm{~N}, 50,36$, and $20 \mathrm{~N}$, respectively [17]. The coefficients of friction between the ground and insole, and between the insole and the encapsulated soft tissue were both assumed 0.6 [18].

\subsection{Model output and data analysis}

The FE simulation was conducted using the commercial FE package, Abaqus (Dassault Systèmes, Vélizy-Villacoublay, France). The predicted maximum von Mises stress of the navicular and cuneiform bones, the articular cartilage of the medial cuneonavicular joint, and the plantar foot regions (heel, midfoot, and metatarsal) were extracted for analysis. The mechanical stress on the bones and the articular cartilage had direct implications to the arthritic condition of the joint and served as the primary outcome. Plan- 
Table 2. Finite element prediction of the 9 configurations of the insole in an $\mathrm{L}_{9}$ orthogonal array

\begin{tabular}{|c|c|c|c|c|c|c|c|c|c|c|}
\hline \multirow{2}{*}{ Trial Number } & \multirow{2}{*}{ Code } & \multirow{2}{*}{$H$} & \multirow{2}{*}{$A$} & \multirow{2}{*}{$E$} & \multicolumn{6}{|c|}{ Finite Element Prediction [MPa] } \\
\cline { 7 - 12 } & & & & & $\mathrm{S}_{\text {cart }}$ & $\mathrm{S}_{\text {navi }}$ & $\mathrm{S}_{\text {cune }}$ & $\mathrm{S}_{\text {P-heel }}$ & $\mathrm{S}_{\text {P-midF }}$ & $\mathrm{S}_{\text {P-MT }}$ \\
\hline 1 & H1A1E1 & 1 & 1 & 1 & 0.156 & 8.40 & 7.28 & 0.086 & 0.064 & 0.072 \\
\hline 2 & H1A2E2 & 1 & 2 & 2 & 0.146 & 8.04 & 6.71 & 0.058 & 0.060 & 0.061 \\
\hline 3 & H1A3E3 & 1 & 3 & 3 & 0.140 & 8.08 & 6.41 & 0.075 & 0.068 & 0.083 \\
\hline 4 & H2A2E3 & 2 & 2 & 3 & 0.137 & 8.59 & 6.79 & 0.062 & 0.060 & 0.057 \\
\hline 5 & H2A3E1 & 2 & 3 & 1 & 0.121 & 7.83 & 4.89 & 0.059 & 0.042 & 0.042 \\
\hline 6 & H2A1E2 & 2 & 1 & 2 & 0.134 & 8.26 & 5.37 & 0.054 & 0.047 & 0.049 \\
\hline 7 & H3A3E2 & 3 & 3 & 2 & 0.118 & 7.31 & 5.02 & 0.057 & 0.046 & 0.055 \\
\hline 8 & H3A1E3 & 3 & 1 & 3 & 0.126 & 7.30 & 5.49 & 0.056 & 0.052 & 0.083 \\
\hline 9 & H3A2E1 & 3 & 2 & 1 & 0.124 & 7.70 & 5.84 & 0.062 & 0.053 & 0.071 \\
\hline
\end{tabular}

$H$ - Arch height, $A$ - Wedge angle, $E$ - Insole stiffness, Maximum von Mises stress of the articular cartilage at the medial cuneonavicular joint $\left(\mathrm{S}_{\text {cart }}\right)$, navicular $\left(\mathrm{S}_{\text {navi }}\right)$, medial cuneiform $\left(\mathrm{S}_{\text {cune }}\right)$, plantar heel region $\left(\mathrm{S}_{\mathrm{P} \text {-heel }}\right)$, plantar midfoot region $\left(\mathrm{S}_{\mathrm{P}-\mathrm{midF}}\right)$, and plantar metatarsal region $\left(\mathrm{S}_{\mathrm{P}-\mathrm{MT}}\right)$.

tar stress has been commonly used as an evaluation metric for orthotics, and may indirectly reflect the support in different plantar foot regions. As shown in Table 2, the effects of the three design factors of insoles $(H, A$, and $E)$ were analyzed using the Taguchi method, in which 9 combinations of design factors using the $\mathrm{L}_{9}$ orthogonal array were sufficient to identify the significance of each design factor instead of the 27 combinations in a full factorial design. The column of an orthogonal array is mutually orthogonal in which for any pair of columns, all combinations of factor levels occur at an equal number of times.

An analysis of variance was performed for the between-group sum of squares to determine the sensitivity of each design factor. The sum of squares (SS) of a design factor is defined as:

$$
\mathrm{SS}=\sum_{i=1}^{3}\left(R_{i}-R_{m}\right)
$$

where $R_{i}$ was the outcome of factor $R$ at the $i$-th level. $R_{m}$ was the balanced overall mean over the entire 9 simulations (i.e., mean of all values of a particular outcome variable).

\subsection{Model validation}

The model was validated by comparing the FE predicted plantar pressure to the measured plantar pressure of the model participant using TPScan (Biomecha, Goyang, Korea) under balanced standing and midstance condition barefoot without the wedge insole. During the midstance condition, the peak plantar pressures of the FE prediction and measurement were $0.12 \mathrm{MPa}$ and $0.13 \mathrm{MPa}$, respectively. During balanced standing, the plantar contact area of the meta- tarsal region of the prediction and measurement were $1784.0 \mathrm{~mm}^{2}$ and $1761.1 \mathrm{~mm}^{2}$, respectively, while those of the heel region were $1948.6 \mathrm{~mm}^{2}$ and 1976.8 $\mathrm{mm}^{2}$. The percentage deviations between the prediction and measurement were less than $10 \%$. We viewed that the FE simulation was validated and adequately reliable.

\section{Results}

The FE predicted peak plantar pressure of the heel and metatarsal regions among the 9 configurations of the insole is listed in Table 2, while the main effect of each design factor at each level is illustrated in Fig. 4.

The von Mises stress distribution of the cartilage among the 9 configurations is illustrated in Fig. 5. Insole with the highest arch and softest material yet medium lateral wedge angle (H3A3E2) produced the lowest cartilage stress at the arthritic joint. However, this selection did not produce the lowest stress for the navicular and medial cuneiform bones. High arch height and stiffness, yet low wedge angle insole (H3A1E3) was required to minimize the navicular stress while the lowest stress was induced by an insole with low arch height, medium level of wedge angle and stiffness (H2A3E1) for the medial cuneiform bone.

An elevated arch height generally decreased the peak plantar stress. Interesting, insoles with a medium level of arch height produced higher peak plantar stress compared to other conditions. Lower wedge angle and softer insole material reduced the peak plantar stress of the heel region, while that of the metatarsal region was relieved by a medium level of wedge angle and insole stiffness. 


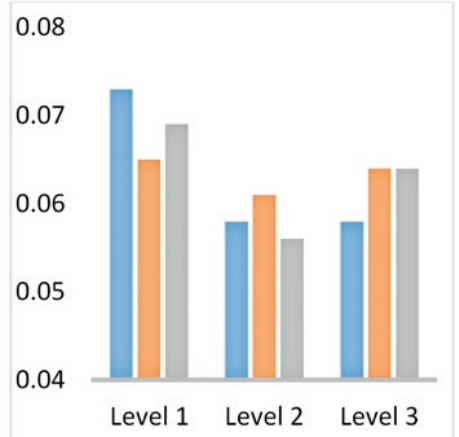

(a)

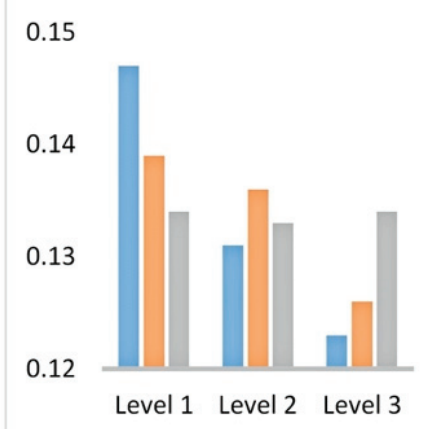

(d)

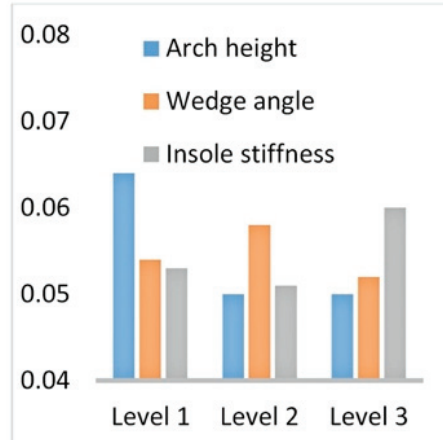

(b)

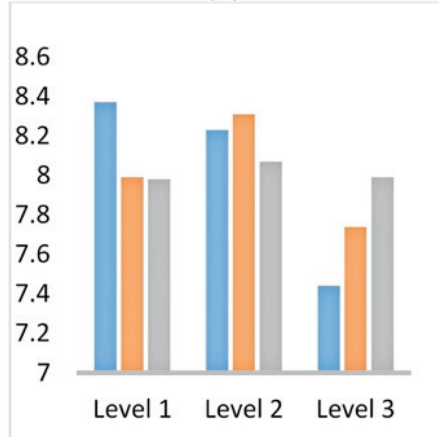

(e)

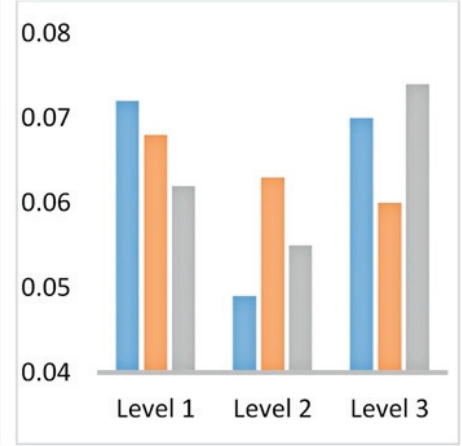

(c)

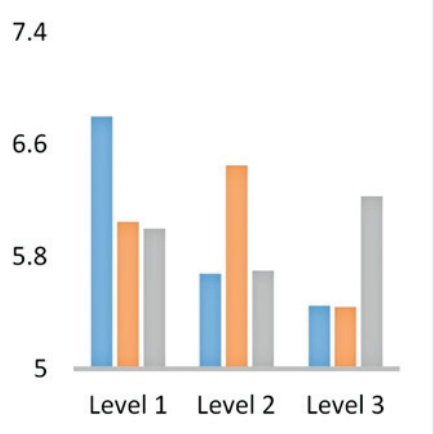

(f)

Fig. 4. The main effect of the three design factors at each level on the predicted maximum von Mises stress [MPa] of the (a) plantar heel, (b) plantar midfoot, (c) plantar metatarsal region, (d) articular cartilage at the medial cuneonavicular joint, (e) navicular and (f) medial cuneiform. The denotation of Level 1 to Level 3 refers to Table 1

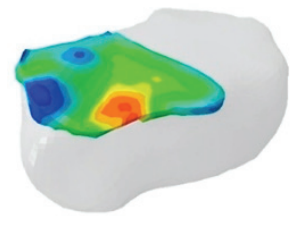

H2A1E2

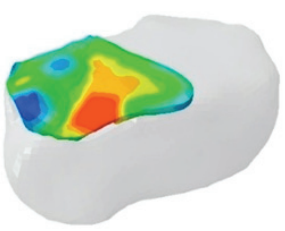

H1A1E1

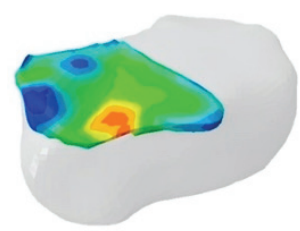

H3A1E3

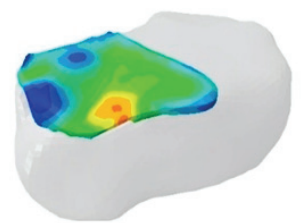

H2A3E1

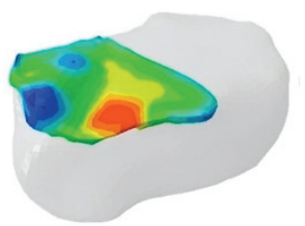

H1A3E3

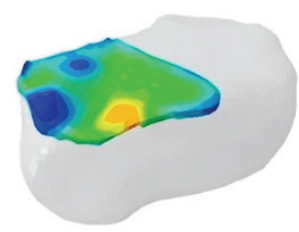

H3A3E2

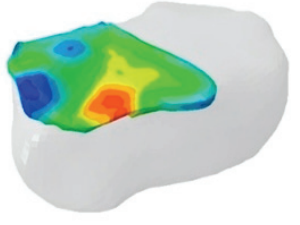

H2A2E3

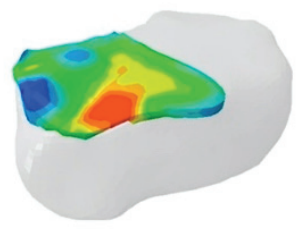

H1A2E2

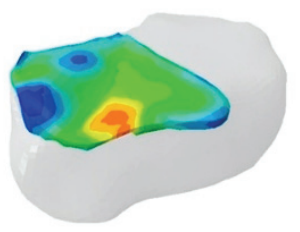

H3A2E1

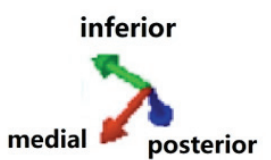

Fig. 5. von Mises stress distribution of the articular cartilage at the medial cuneonavicular joint.

The illustration shows the articular cartilage on the navicular bone at a view from the dorsolateral perspective. The settings of the code refer to Table 3 
Table 3. Sum of squares of the FE prediction for the 3-factor, 3-level fractional factorial analysis

\begin{tabular}{|c|c|c|c|c|c|c|}
\hline \multirow{2}{*}{ Factor } & \multicolumn{7}{|c|}{ Sum of Squares $\left(\times 10^{-4} \mathrm{MPa}^{2}\right)$} \\
\cline { 2 - 7 } & $\mathrm{S}_{\text {cart }}$ & $\mathrm{S}_{\text {navi }}$ & $\mathrm{S}_{\text {cune }}$ & $\mathrm{S}_{\text {P-heel }}$ & $\mathrm{S}_{\text {P-midF }}$ & $\mathrm{S}_{\text {P-MT }}$ \\
\hline $\mathrm{H}$ & 3.17 & 3.898 .7 & 10.413 .0 & 0.83 & 3.50 & 0.01 \\
\hline $\mathrm{A}$ & 1.43 & 709.9 & 5.138 .1 & 0.11 & 2.36 & 0.82 \\
\hline $\mathrm{E}$ & 6.43 & 86.5 & 1.414 .3 & 5.03 & 2.64 & 5.03 \\
\hline
\end{tabular}

The importance level for each insole design factor was illustrated by the sum of squares as shown in Table 3 . The stress of the articular cartilage was proven primarily contributed by the insole stiffness. Similarly, the selection of insole stiffness was found predominantly affected the peak plantar stress on both heel and metatarsal regions and the strength of the contributor was much higher than the other factors. Arch height was the second important influencer to the peak stress of the heel region whilst that of the metatarsal region was the wedge angle. Besides, for the plantar stress of the midfoot, the arch height was the primary contributor despite that the difference in the level of importance among the design factors was relatively small. On the other hand, the stress of the navicular and medial cuneiform was least affected by the change of insole stiffness but largely influenced by the arch height.

\section{Discussion}

In this study, we reconstructed and validated a three-dimensional FE model of the foot and ankle to evaluate 9 different configurations of insole designs with 3 design factors. Our prediction showed that insole with a high arch, low stiffness, and medium level of lateral wedge angle (i.e., $H=30 \mathrm{~mm} ; A=5^{\circ} ; E=1.0 \mathrm{MPa}$ ) could minimize the stress of the articular cartilage at the arthritic joint and could be beneficial to patients with midfoot arthritis. Insole stiffness was the dominant contributor to cartilage stress. The primary goal of orthosis for midfoot arthritis patients was to stabilize the midfoot joint that facilitates functions. The high arch of the insole suggested in this study could provide sufficient support to the medial midfoot regions. While midfoot arthritis was often associated with acquired flatfoot deformity, the high but soft foot arch support could prevent the collapse of the medial longitudinal arch and the deterioration of the flatfoot deformity yet compromising comfort. The elevation of the wedge angle in the midfoot may produce an interaction effect with the arch height to maintain the medial longitudinal arch and midfoot stability that requires further investigation. In fact, research often focused on the use of a lateral wedged insole to treat knee osteoarthritis and the wedge was found to reduce the knee adduction moment and increase the subtalar joint valgus moment [19].

Nevertheless, the optimal combination of the insole to minimize cartilage stress did not agree with the solutions suggested by the secondary outcomes, for the reason that the bone stress not only reflects the arthritic condition of the joint but also manifests other compensatory conditions for both bones and soft tissues. The change in foot intersegmental kinematics could also taut or relieve the tension of some ligaments and muscles that may alter the bone stress at the insertion points. In our study, the selection of design factors to minimize stress on the navicular and medial cuneiform was totally different (H3A1E3 vs H2A3E1). Instead of the maximum stress of the whole bone, targeting stress at particular regions of the bone, such as at the muscle attachment point or the particular joint interface, may provide additional insights to explain the differences.

Foot orthoses often endeavored to minimize peak plantar stress since high stress was often linked to pain and discomfort of the foot [20], [21]. While there was a lack of studies on the validated pressure or stress threshold of pain and discomfort, heading for lower plantar stress may not necessarily the goal for midfoot arthritis patients since they require enough midfoot support and stability. Indeed, there was always a trade-off between the level of stress and the sufficiency of supporting load. Chapman et al. [22] evaluated the Functional Foot Orthosis (FFO) which consisted of a heel cup, arch support, and a metatarsal dome. Their study showed that midfoot arthritis patients experienced a reduction in pain and functional improvement in FFO that were powered by the increase in supporting force and contact area under the midfoot region [22]. In our case, an increase in peak plantar stress of the midfoot was facilitated by a medium arch height and wedge angle, and stiffest insole material, notwithstanding the combination neither reduced the peak plantar stress of other foot regions nor was consistent with the combination that minimized the stress of the articular cartilage. 
A few investigations on total contact insoles have been investigated using FE models despite different purposes. Majority of studies designs insoles to redistribute the plantar pressure to prevent tissue breakdown for diabetic neuropathy patients. For example, Chen et al. [20] evaluated the influence of different insole materials on the reduction of peak plantar pressure. Cheung et al. [8] implemented a Taguchi approach to determine the optimal combination of arch type, insole, and outsole material and thickness in designing the total contact insoles. Other applications included the relief of plantar fascia stress, forefoot pain [21], and accommodation to foot deformities [23].

It is controversial whether the model geometry of the plantar fascia shall be simplified. The geometry reconstruction of plantar fascia was traditionally simplified by truss units and supported by some validation experiments. These simulations predominantly targeted on the influence of plantar fascia release which were validated by strain measurement on plantar fascia using cadaveric experiments [15], [24]. Recently, attempts have been made to reconstruct a three-dimensional anatomically detailed plantar fascia [21], [25]. The assigned material property of the detailed model failed to address the tension-only and anisotropy characteristics of the fascia bundles. In addition, direct validation onto the plantar fascia using the detailed model reconstruction approach has been lacking. The simulation may overestimate the plantar fascia stress under bending or twisting condition. We therefore decided to adopt the truss approach which was better verified previously.

\section{Conclusions}

There were some limitations to this study. We adopted a single-subject approach in this study that hindered the external validity and generalizability of the finding. In fact, it has been a problem faced by simulation studies using finite element foot model because of the strenuous work involved in reconstructing the complex foot and ankle structure [7]. In addition, a patient-specific boundary and loading condition was often lacking which could be necessary to improve model prediction accuracy [7]. In addition, we filled up the articular joint space with the encapsulated soft tissue and assumed that the material of the encapsulated soft tissue took up the role of the articular cartilage, whereas the geometry of the cartilage at the arthritic site was reconstructed for more detailed analysis. The discrepancy of model reconstruction methods among the cartilage may potentially shift the load transfer proportion among metatarsal columns. Besides simplifications inherited by the model reconstruction and material property assignment, the simulation was confined to a particular loading case at midstance instant. Simulation for the whole walking stance could facilitate a complete loading profile for analysis. Besides, we assumed that the boundary and loading conditions, as well as the muscle forces of midfoot arthritis patients were similar to that of normal individuals. A patient-specific kinematic and muscle force profile is necessary to improve the accuracy of the prediction. While computational models are normally developed to simulate conditions that are impractical or difficult to be addressed experimentally [6], our validation experiment considered the barefoot condition without the wedge insole. Measurement of plantar pressure with the wedge insole could improve the reliability of the simulation. Future studies can consider investigating the influence of insole stiffness and wedge angle at different regions of the insole.

\section{Acknowledgement}

The research was funded by the project of the academic leader of health system in Pudong New Area, Shanghai (PWRd2019-05) and Shanghai Municipal Health Bureau (201840137).

\section{References}

[1] Verhoeven N., VAndeputte G., Midfoot arthritis: diagnosis and treatment, Foot and Ankle Surgery, 2012, 18 (4), 255-262.

[2] KuRUP H., VASUKUTTY N., Midfoot arthritis-current concepts review, Journal of Clinical Orthopaedics and Trauma, 2020, 11 (3), 399-405.

[3] Rao S., Baumhauer J.F., TOME J., NAwoczenSKi D.A., Orthoses alter in vivo segmental foot kinematics during walking in patients with midfoot arthritis, Archives of Physical Medicine and Rehabilitation, 2010, 91 (4), 608-614.

[4] Rao S., Baumhauer J.F., Becica L., Nawoczenski D.A., Shoe inserts alter plantar loading and function in patients with midfoot arthritis, Journal of Orthopaedic and Sports Physical Therapy, 2009, 39 (7), 522-531.

[5] Halstead J., Chapman G.J., Gray J.C., Grainger A.J., Brown S., WiLkins R.A. et al., Foot orthoses in the treatment of symptomatic midfoot osteoarthritis using clinical and biomechanical outcomes: a randomised feasibility study, Clinical Rheumatology, 2016, 35 (4), 987-996.

[6] Morales-Orcajo E., Bayod J., De Las Casas E.B., Computational foot modeling: scope and applications, Archives of Computational Methods in Engineering, 2016, 23 (3), 389-416.

[7] Wang Y., Wong D.W.-C., Zhang M., Computational models of the foot and ankle for pathomechanics and clinical applications: a review, Annals of Biomedical Engineering, 2016, 44 (1), 213-221. 
[8] Cheung J.T.-M., Zhang M., Parametric design of pressure-relieving foot orthosis using statistics-based finite element method, Medical Engineering and Physics, 2008, 30 (3), 269-277.

[9] Wong D.W.-C., Wang Y., Leung A.K.-L., Yang M., ZHANG M., Finite element simulation on posterior tibial tendinopathy: load transfer alteration and implications to the onset of pes planus, Clinical Biomechanics, 2018, 51, 10-16.

[10] NaKamura S., Crowninshield R., CoOper R., An analysis of soft tissue loading in the foot - a preliminary report, Bulletin of Prosthetics Research, 1981, 10, 27-34.

[11] Athanasiou K., Liu G., LAVERY L., LANCTOt D., SCHENCK J.R., Biomechanical topography of human articular cartilage in the first metatarsophalangeal joint, Clinical Orthopaedics and Related Research, 1998, (348), 269-281.

[12] Lemmon D., Shiang T.-Y., Hashmi A., Ulbrecht J.S., CAVANAGH P.R., The effect of insoles in therapeutic footwear - a finite element approach, Journal of Biomechanics, 1997, 30 (6), 615-620.

[13] Siegler S., Block J., SCHNECK C.D., The mechanical characteristics of the collateral ligaments of the human ankle joint, Foot and Ankle, 1988, 8 (5), 234-242.

[14] Wright D., RenNELS D., A study of the elastic properties of plantar fascia, Journal of Bone and Joint Surgery, 1964, 46 (3), 482-492.

[15] Cheung J.T.-M., Zhang M., An K.-N., Effect of Achilles tendon loading on plantar fascia tension in the standing foot, Clinical Biomechanics, 2006, 21 (2), 194-203.

[16] Chen Y.-N., Chang C.-W., Li C.-T., Chang C.-H., Lin C.-F., Finite element analysis of plantar fascia during walking: a quasi-static simulation, Foot and Ankle International, 2015, 36 (1), 90-97.
[17] Arnold E.M., Ward S.R., Lieber R.L., Delp S.L., A model of the lower limb for analysis of human movement, Annals of Biomedical Engineering, 2010, 38 (2), 269-279.

[18] Zhang M., MAK A., In vivo friction properties of human skin, Prosthetics and Orthotics International, 1999, 23 (2), 135-141.

[19] Kakihana W., AKai M., Nakazawa K., Takashima T., NAITO K., TORII S., Effects of laterally wedged insoles on knee and subtalar joint moments, Archives of Physical Medicine and Rehabilitation, 2005, 86 (7), 1465-1471.

[20] CHen W.-P., Ju C.-W., TANG F.-T., Effects of total contact insoles on the plantar stress redistribution: a finite element analysis, Clinical Biomechanics, 2003, 18 (6), S17-S24.

[21] Chen W.-M., LeE S.-J., LeE P.V.S., Plantar pressure relief under the metatarsal heads-Therapeutic insole design using three-dimensional finite element model of the foot, Journal of Biomechanics, 2015, 48 (4), 659-665.

[22] Chapman G.J., Halstead J., Redmond A.C., Comparability of off the shelf foot orthoses in the redistribution of forces in midfoot osteoarthritis patients, Gait and Posture, 2016, 49, 235-240.

[23] Anggoro P., Saputra E., TAuviqirrahman M., Jamari J., BAYUSENO A., A 3-dimensional finite element analysis of the insole shoe orthotic for foot deformities, International Journal of Applied Engineering Research, 2017, 12 (15), 5254-3260.

[24] Liang J., Yang Y., Yu G., Niu W., Wang Y., Deformation and stress distribution of the human foot after plantar ligaments release: A cadaveric study and finite element analysis, Science China Life Sciences, 2011, 54 (3), 267-271.

[25] Chen T.L.-W., Wong D.W.-C., Wang Y., Lin J., Zhang M., Foot arch deformation and plantar fascia loading during running with rearfoot strike and forefoot strike: a dynamic finite element analysis, Journal of Biomechanics, 2019, 83, 260-272. 